\title{
Hochkonzentriertes Insulin seit einem Jahr verfügbar
}

Seit einem Jahr steht in Deutschland Insulin glargin U300 (300E/ml, Toujeo $\left.{ }^{\circledR}\right)$ zur Behandlung von Menschen mit Typ1- und Typ-2-Diabetes zur Verfügung. Insulin glargin U300 (Toujeo ${ }^{\circledR}$ ) enthält $300 \mathrm{E} / \mathrm{ml}$ und benötigt somit für die gleiche Anzahl an Einheiten nur ein Drittel des Volumens des häufig verwendeten Insulin glargin U100 (Lantus ${ }^{\circledR}$ ). „Deshalb bildet Insulin glargin U300 im subkutanen Fettgewebe ein Depot, das nur eine halb so große Oberfläche hat wie bisher; es wird langsamer und gleichmäßiger freigesetzt und hat ein noch flacheres Wirkprofil“, erklärte Prof. Dr. Robert Ritzel, München, in Berlin. „Hier genügt wirklich für alle Patienten eine einmalige Injektion pro Tag.“

Eine von Ritzel auf dem Diabeteskongress in Berlin vorgestellte Metaanalyse der Studien EDITION 1, 2 und 3 erfasst die Wirkung und Sicherheit von Insulin glargin U300 bei Menschen mit Typ-2-Diabetes und unterschiedlichen
Vortherapien (auf Ebene der einzelnen Patientendaten) über ein Jahr. Darin hat sich die effektive Blutzuckersenkung unter Insulin glargin U300 bestätigt: Nach insgesamt 12 Monaten war der Effekt auf den $\mathrm{HbA}_{1 \mathrm{c}}$-Wert unter Insulin glargin U300 sogar leicht, aber signifikant stärker als unter U100. Nach wie vor wurden nachts und über den gesamten Tag unter Insulin glargin U300 weniger schwere und bestätigte Hypoglykämien beobachtet; zudem war die Gewichtsentwicklung unter Insulin glargin U300 etwas günstiger [1].

Von Insulin glargin U300 können viele Patienten profitieren

Mit Insulin glargin U300 behandeln würde Ritzel Patienten

- mit Typ-1- oder Typ-2-Diabetes, die neu auf ein Basalinsulin eingestellt werden,

- mit wiederholten oder nächtlichen Hypoglykämien,
- mit Furcht vor Hypoglykämien oder vor Gewichtszunahme und

- mit hohem Alter oder bestehender Nierenfunktionsstörung (wegen der besonderen Notwendigkeit einer gleichmäßigen Wirkung des Basalinsulins).

Der Experte verwies zudem auf die hohe zeitliche Unabhängigkeit der Patienten unter Insulin glargin U300: Sie können zu Therapiebeginn einen Injektionszeitpunkt frei wählen und haben dann ein flexibles Zeitfenster von \pm 3 Stunden; Patienten, die darauf angewiesen sind, sieht Ritzel ebenfalls als Kandidaten für die Therapie.

Simone Reisdorf, Erfurt

\section{Literatur}

1 Ritzel R et al. Diabeteskongress Mai 2016, Poster \# 50

Quelle: Fachpressekonferenz „Herzlichen Glückwunsch - 1 Jahr Insulin glargin U300“ am 04.05.2016 in Berlin. Veranstalter: Sanofi

\section{Gute Option auch in Extremsituationen wie Ramadan}

Chronisch kranke Menschen müssen nicht am Ramadan teilnehmen - der nächste findet vom 6. Juni bis 4. Juli 2016 statt -, aber rund $80 \%$ der muslimischen Diabetiker tun dies, sagte Prof. Werner Kern aus Ulm. In dieser Zeit wird von Sonnenaufgang bis -untergang gefastet, sodass Hypoglykämien begünstigt werden. Vor dem Schlafengehen gibt es dann eine üppige Mahlzeit, die zu einem deutlichen Anstieg der Blutglukosewerte führt. Insbesondere eine Therapie mit Mischinsulinen ist dann problematisch, sagte Kern. Vorteilhaft sind hingegen GLP-1-Analoga, deren Wirkung Glukose-abhängig ist. „Wenn normale Blutzuckerwerte erreicht sind, hören die Effekte auf", sagte der Diabetologe.

Die Vorteile gerade in Extrembedingungen wurden in der LIRA-Ramadan Studie bei insgesamt 343 Typ-2-Diabetikern belegt, die beabsichtigten, während des Ramadan zu fasten. Die Studienteilnehmer waren auf eine Kombination von
Metformin und Sulfonylharnstoff (SH) eingestellt; die Hälfte wurde dann randomisiert mindestens 10 Wochen vor Beginn des Ramadan auf Metformin plus Liraglutid $1 \mathrm{mg}$ (Victoza ${ }^{\circledR}$ ) umgestellt. Die Follow-up-Phase dauerte 33 Wochen. Anpassungen der SH-Dosis bzw. des Verabreichungszeitpunkts waren erlaubt.

Bereits bis zum Beginn des Fastenmonats verringerte sich der $\mathrm{HbA}_{1 \mathrm{c}}$-Wert in der Liraglutid-Gruppe stärker als in der SH-Gruppe (1,1 vs. -0,4\%). Am Ende des Ramadan betrugen die $\mathrm{HbA}_{1 \mathrm{c}}$-Unterschiede zwischen beiden Gruppen rund 0,6\% $(1,24$ vs. $0,65 \%)$ [1]. Den $\mathrm{HbA}_{1 \mathrm{c}}$-Zielwert $<7 \%$ erreichten $57 \%$ der Patienten der Liraglutid-Gruppe vs. 26\% der SH-Gruppe. Trotz der bereits besseren Blutzuckerkontrolle zu Beginn des Ramadan nahm die Fructosamin-Konzentration während des Fastenmonats - ein Marker für die kurzfristige Blutzuckerkontrolle - in beiden Gruppen in ähnlichem Maße ab und war das Risiko für symptomatische Hypogly- kämien unter Luraglutid deutlich geringer (2 vs. $11 \%$ ). Zudem traten unter dem GLP-1-Analogon während der Nahrungskarenzphase keine bestätigten nächtlichen Hypoglykämien auf im Vergleich zu 3 Episoden unter SH, berichtete Kern. Das Körpergewicht der Patienten verringerte sich in der Liraglutid-Gruppe während des Ramadan um rund $1,4 \mathrm{~kg}$ und im gesamten Studienverlauf um mehr als $5 \mathrm{~kg}$ im Vergleich zu 0,9 kg bzw. 1,2 kg in der SH-Gruppe. Liraglutid wurde von den meisten Patienten auch während des Ramadan gut vertragen, so Kern.

Roland Fath, Hamburg

\section{Literatur \\ 1 Kern W et al. DDG 2016, Berlin, Poster 41}

Quelle: Pressegespräch „Belastungsprobe für die Diabetestherapie: Liraglutid als Option bei stark unregelmäßiger Nahrungsaufnahme im Rahmen des Ramadan?“, 5. Mai 2016, Berlin. Veranstalter: Novo Nordisk Pharma GmbH, Mainz 